\title{
The Comparison of 3D and 2D Measurement Techniques Used for the Analysis of Vehicle Deformation
}

\author{
Pavlína Moravcová ${ }^{1,2} \mathbb{C}^{\mathrm{a}}$, Kateřina Bucsuházy ${ }^{1,2} \mathbb{E}^{\mathrm{b}}$, Robert Zůvala ${ }^{2} \mathbb{C}^{\mathrm{c}}$, Martin Bilík ${ }^{1} \mathbb{C}^{\mathrm{d}}$ \\ and Albert Bradác ${ }^{1}{ }^{\text {iD }}$ \\ ${ }^{I}$ Institute of Forensic Engineering, Brno University of Technology, Czech Republic \\ ${ }^{2}$ Transport Research Centre, Brno, Czech Republic
}

Keywords: Vehicle, Deformation, 2D, 3D, Accident Analysis, Laser Scan.

Abstract: As one of the main assumptions for the accident analysis has been detailed information about vehicle deformation. The precise deformation depth allows to quantify deformation energy and related impact speed. The aim of this paper has been the comparison of two selected methods used for the determination of deformation depth. For the purpose of this paper were selected top-view photography as basic and cheap method and 3D scanning as modern and advanced method. Different vehicles and 2 basic types of damage frontal and side impact - were chosen for the analysis. Also, the different range of vehicle deformation depth were selected. On the basis of obtained results is possible to determine the applicability of these methods, their advantages and limitations.

\section{INTRODUCTION}

Vehicle damage analysis (the vehicle deformation respectively) has been one of the main assumptions and inputs for the subsequent accident analysis (speed calculation, determination of impact configuration, etc.). The accident reconstruction based on energy conservation principle was described by e.g. (Campbell, 1974 or McHenry, 1986). In the Czech Republic, the accident documentation and subsequent accident analysis is conducted separately. The documentation is usually carried out by Police immediately after the accident occurrence at the accident place. For the accident technical cause determination (which usually involves vehicle speed calculation) is usually required forensic expert. The accuracy of the accident analysis is dependent on the accuracy of the input data.

For the deformation measurement could be used a variety of methods - the simplest is manual measurement using measurement tape, deformation jigs or grids (Brown, 1987). A detailed measurement methodology using deformation jigs has been published by e.g. Tumbas. For the deformation measurement could be also used measurement from photographs (especially top-view photo) as the lowcost method for accident documentation. However, photographs are sometimes unable to capture the extent of damage. It is sometimes necessary to combine top-view photo with photographs from different angles, because some of the deformation can be hidden under the hood (Boddorff, 1990). As the modern methods are for documentation of vehicle deformation used e.g. geodetic total stations, quadrocopter, photogrammetry and especially $3 \mathrm{D}$ scanning.

For some types of vehicle damage, only the 2D representation (e.g. photography) is sufficient, some types of vehicle deformation require detailed 3D imaging (scanners, photogrammetry, etc.) (Massa, Barrete; 1998). The accuracy of selected methods for accident documentation was studied by a number of previous studies. Comeau (1996) compared the manual measurement with total station measurement. The accuracy has been significantly influenced by initial settings, especially accuracy of the manual

(Di) https://orcid.org/0000-0002-9005-703X

b (iD https://orcid.org/ 0000-0003-1247-6148

c(i) https://orcid.org/ 0000-0003-2038-7292

d (D) https://orcid.org/ 0000-0003-3796-4658

e(i) https://orcid.org/ 0000-0001-7587-1474 
measurement has been influenced by precision of the investigator. The maximum deviation between manual measurement and total station was $3 \mathrm{~cm}$ in the maximum deformation region. The top view photography was used by Boddorff (Boddorf, 1990), the average measurement deviation ranged from 6 to $18 \%$. Significant differences could be influenced e.g. by the hood of the vehicle which overlaps some of the damaged measured parts. Also, the three-dimensional imaging as photogrammetry and its accuracy has been studied e.g. by (Tumbas, 1994, Fenton and Kerr, 1997; Neale, 2004). Using high resolution digital images in conjunction with control points and constraints from three-dimensional data has proven to be an effective way to reconstruct vehicle damage (Buck, 2007; Karczewsk, 2019). Nowadays, especially high-resolution laser scanning is used for the vehicle damage documentation (Callahan, 2012; Kang, 2017). Laser scanning has been also widely used for traffic accident documentation (Kamnik, 2020) or 3D human body analysis. Laser scanning allows to detect traces which are unrecognizable at the accident scene. Subsequent analysis also allows to analyse traces from different perspective (Harrington, 2018). In terms of both financial and time requirements, only photographs are still available in most cases. The aim of this study has been the comparison of two selected measurement methods 2D (top-view photographs) and 3D (laser scanners) and determine the applicability of these methods in different types of impacts (frontal and side impacts).

\section{METHODS}

For the purpose of this study was selected two methods: photography (top-view) as the widely used, simplest and cheapest method and 3D laser scanning as one of the most modern method. The vehicle deformation analysis using 3D scanning has been performed by comparison of the damaged vehicle scan with the similar undamaged vehicle model (figure 2). 3D view allows to select the maximum area of the vehicle damage (figure 1). The measurement from the 3D scanning was realized using 2D cut in the level of the maximum deformation depth. As the software tool for the analysis of vehicle scans were used Geomagic Control.

For the measurement of the deformation depth using the top-view photography were used the photo of damage vehicle and undamaged vehicle model from the software Autoview (figure 3).

Predefined measurement points were defined on each of the vehicles. The measurement points were distributed equally by every $10 \mathrm{~cm}$. The predefined points allowed direct point-by-point comparison of both used methods. The number of measured points on each vehicle varied depending on the deformation magnitude.

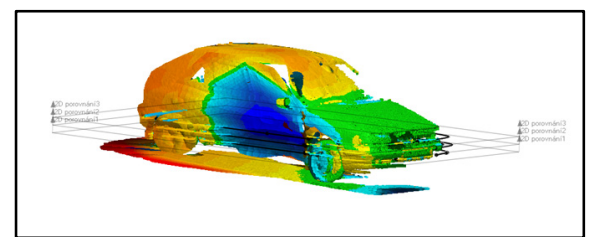

Figure 1: Analysis of 3D scans at maximum deformation depth.

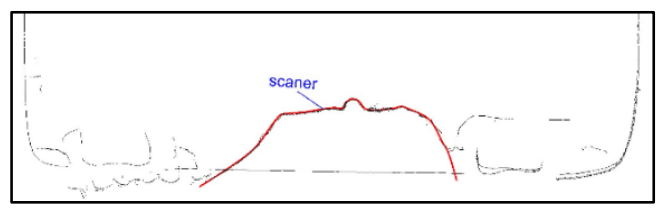

Figure 2: Analysis of 3D scans at maximum deformation depth.

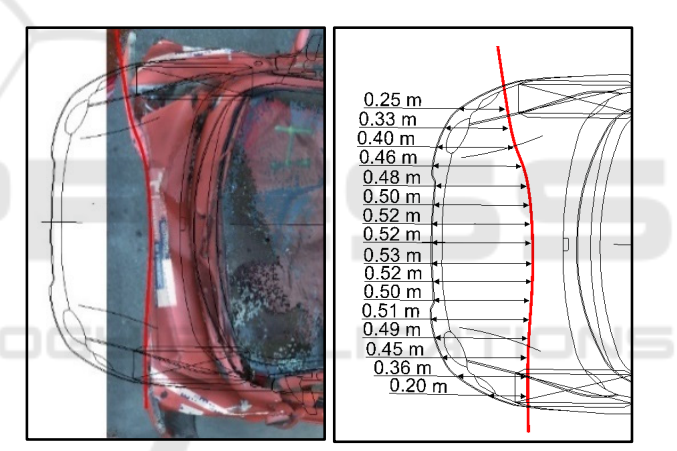

Figure 3: Analysis of photography - top-view.

For the analysis of the methods accuracy were used 3 frontal and 3 side impacts. Selected vehicles have not identical characteristics as e.g. vehicle body stiffness, there has been not common feature. Vehicles were selected on the basis of their deformation in order to compare selected methods over a wide range of deformation depth values.

\section{RESULTS}

\subsection{Frontal Impacts}

Figure 4, 5, 6, 7, 8, 9 show the obtained results - the deformation depth in predefined measurement points on the vehicle front. Figure 4 shows the deformation depth measurement using the top-view photography (left) and 3D scan (right) of the Skoda Karoq vehicle 
(F1). The vehicle front has been damaged over the almost entire width - the front bumper, bonnet, longitudinal beams including damage of the vehicle deformation elements. The maximum deformation depth determined using the top-view photography was $21 \mathrm{~cm}$, minimum about $3 \mathrm{~cm}$. After a threedimensional analysis of the maximum damage area, a 2D cut was made at the height of $55 \mathrm{~cm}$. The maximum deformation depth determined using the scan was $12 \mathrm{~cm}$, minimum similarly as using topview photo about $3 \mathrm{~cm}$.

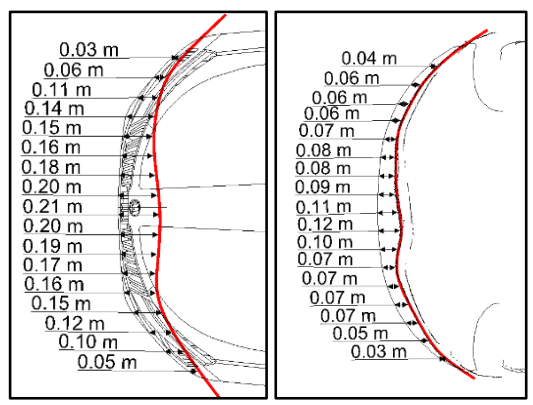

Figure 4: Skoda Karoq frontal impact - top-view (left) vs. scanner (right).

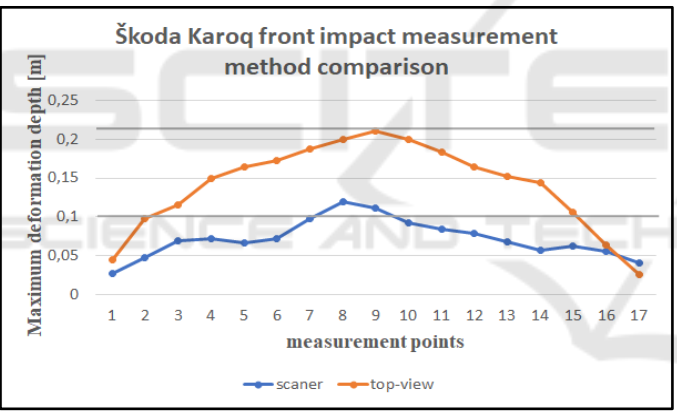

Figure 5: Skoda Karoq front impact measurement method comparison.

Comparison of the measured deformation depth value using the two selected methods demonstrates a considerable difference of the obtained data. Differences of the deformation depth could be influenced mainly by the quality of the top-view photography - e.g. taking photography at the wrong angle which can subsequently distort the deformation analysis.

Figure 6 shows full-width deformation of the Fiat Multipla frontal part (F2). Compared to the previous case study, the deformation magnitude was larger the front beams at the both sides of vehicle were damaged.

The maximum deformation depth determined using the top-view photography was $53 \mathrm{~cm}$, minimum about $2 \mathrm{~cm}$. After a three-dimensional analysis of the maximum damage area, a 2D cut was made at the height of $50 \mathrm{~cm}$. The maximum deformation depth determined using the vehicle scans was $70 \mathrm{~cm}$, minimum about $6 \mathrm{~cm}$.

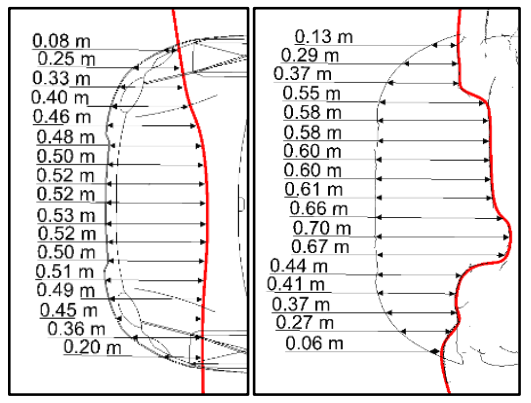

Figure 6: Fiat Multipla frontal impact - top-view (left) vs. scanner (right).

While in the previous case, the deformation depth values were small and the comparison of the selected methods showed significant differences, in this case the comparison of the selected methods shows only small differences. Larger differences of these individual methods have been caused by the covering of the deformation with the vehicle bonnet or other vehicle parts (bumper, etc.).

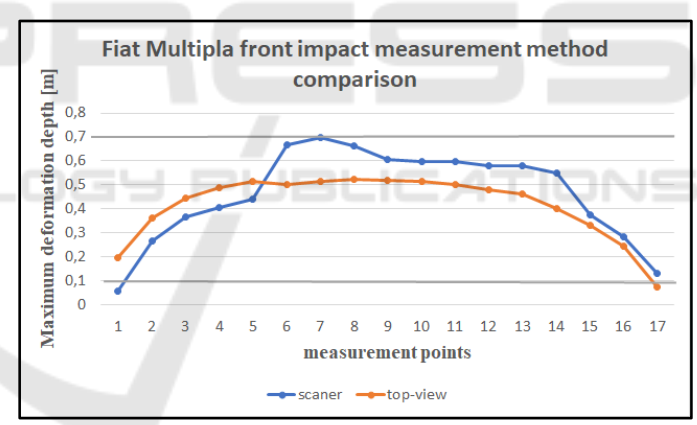

Figure 7: Fiat frontal impact measurement method comparison.

The following frontal collision of the Skoda Felicia (hereinafter referred to as F3) is the most prominent of the presented collisions. There was a complete destruction of the front side of the vehicle, including the front cross member, the left side beam and significantly damaged front axle.

In case of measuring the deformation depth using the top-view (Figure 8), the maximum deformation is $1,13 \mathrm{~m}$ and minimum is $13 \mathrm{~cm}$. With the help of threedimensional analysis, the cut was made in a $50 \mathrm{~cm}$ height, with a maximum deformation about $1,27 \mathrm{~m}$ and minimum about $4 \mathrm{~cm}$. 

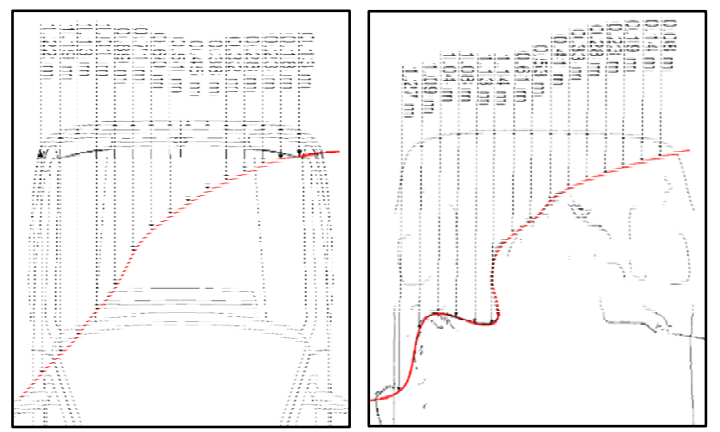

Figure 8: Skoda Felicia frontal impact - top-view (left) vs. scanner (right).

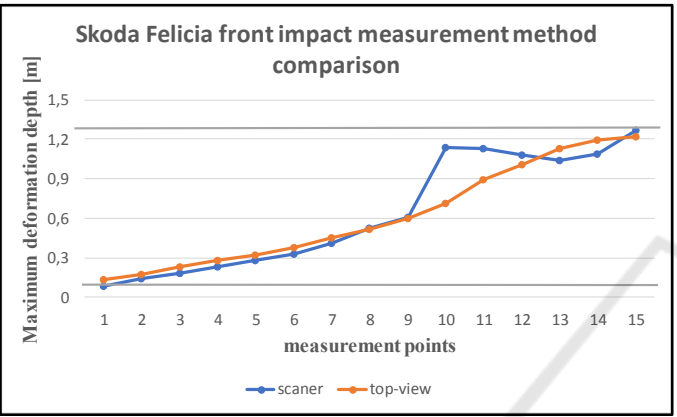

Figure 9: Skoda Felicia front impact measurement method comparison.

The graph (figure 9) shows us that even if such a significant deformation of frontal vehicle part occurs, the deviations are quite minimal. Deviations in measurement in this case occurs due to overlap of the deformation by the windscreen. In case of using combination of methods (e.g. top-view photography and photographs from different angles using of levelling meter), this deviation could be completely eliminated.

\subsection{Side Impacts}

The problematics of deformation measurements in the side collisions is very actual. For the comparison of selected methods were chosen three collision with different character of deformation. Figures 10, 11, 12, $13,14,14$ show the results for the measurement points of the vehicles side.

First case shows side impact of a Skoda Kodiaq (hereinafter referred to as S1). The deformation occurs on the left side of the vehicle, between the A and $\mathrm{C}$ pillars. The left front and rear doors were damaged and the B pillar was slightly deformed.

Figure 10 (up) shows the measurement of the deformation depth using the top-view, where the maximum deformation was about $10 \mathrm{~cm}$ and the minimum was about $4 \mathrm{~cm}$. In three-dimensional analysis, the maximum deformation section was measured at a height of $55 \mathrm{~cm}$ from the ground (Figure 10 - down). The maximum deformation of that section was about $14 \mathrm{~cm}$ and the minimum about $4 \mathrm{~cm}$.

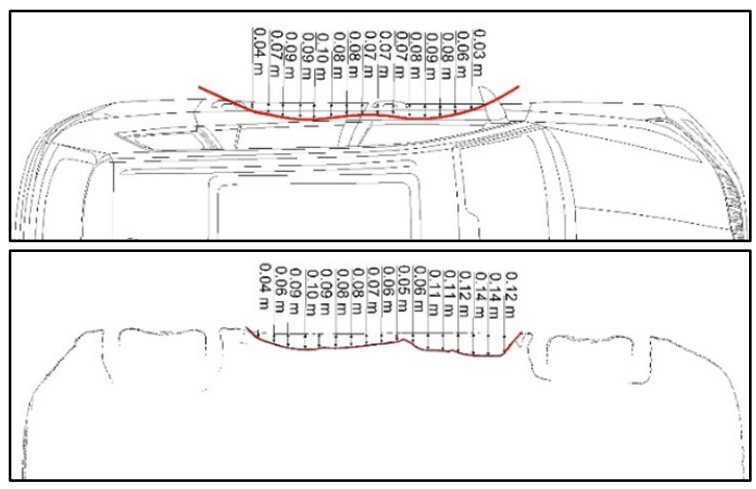

Figure 10: Skoda Kodiaq side impact - top-view (up) vs. scanner (down).

In the graph (figure 11) below could be observed that in case of deformation up to $10 \mathrm{~cm}$ the deviations are not significant, but above $10 \mathrm{~cm}$ the deviation is already visible, even if it is not a significant deformation.

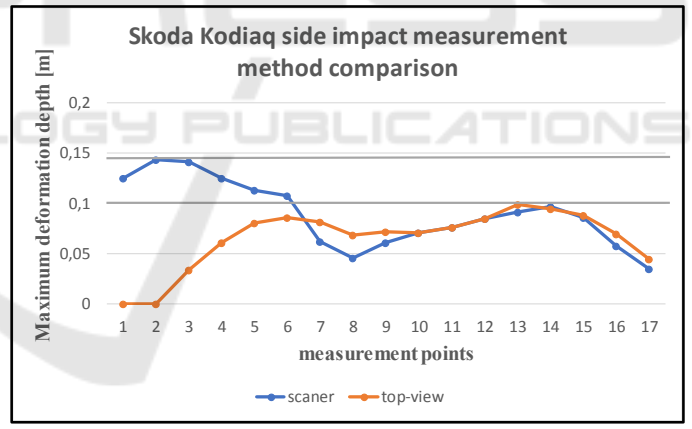

Figure 11: Skoda Kodiaq side impact measurement method comparison.

Another example of a side impact is presented in the following figure. This is the side impact of the Voyager Chrysler (S2). The deformation produced by this impact was already greater than in the previous example. The deformation depth of the S2 collision using the top-view is shown in Figure 12, where the maximum deformation was about $16 \mathrm{~cm}$ and the minimum about $2 \mathrm{~cm}$. In a three-dimensional analysis, the maximum deformation section was at a height of $60 \mathrm{~cm}$ (Figure 12 - down). The maximum deformation was about $27 \mathrm{~cm}$ and the minimum about $3 \mathrm{~cm}$. 


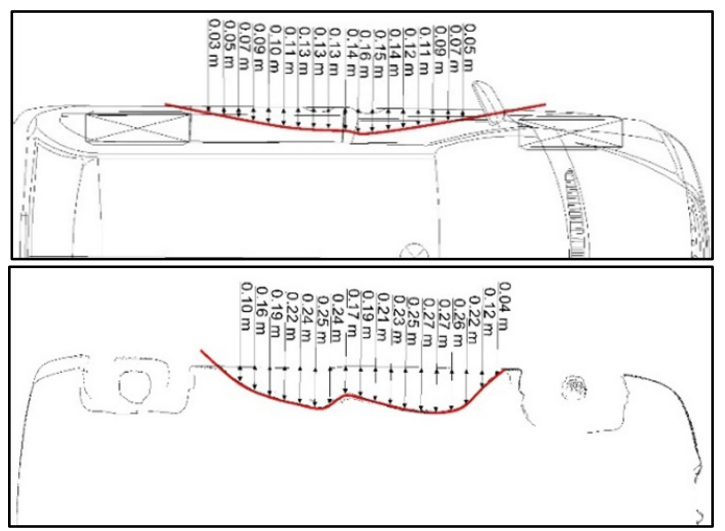

Figure 12: Chrysler Voyager side impact - top-view (up) vs. scanner (down).

The following graph (figure 13) shows similarly as in previous case study not significant deviation in case of deformation up to $10 \mathrm{~cm}$. A significant change occurs in the case of more extent side deformation, such a large deformation may not be apparent from the photograph. As can be seen e.g. in case of maximum deformation. When using a scanner, maximum deformation was measured about $27 \mathrm{~cm}$. Using the top-view photograph at the same point there was the depth of deformation only about $12 \mathrm{~cm}$.

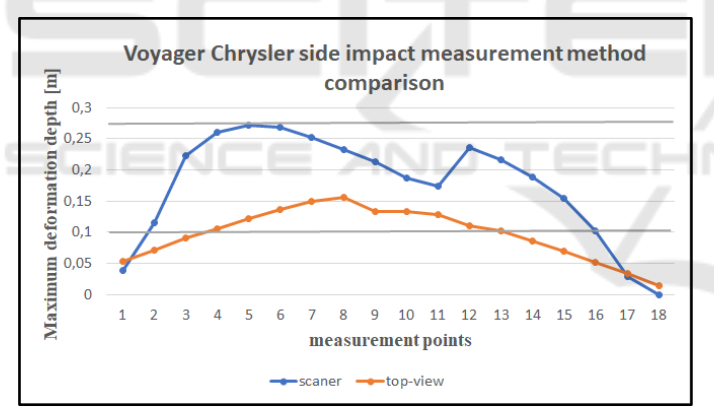

Figure 13: Voyager Chrysler side impact measurement method comparison.

The last example of a side impact is the Skoda Felicia (hereinafter referred to as S3). The deformation of the $\mathrm{S} 3$ impact can be quantified as the most significant of the side impact measurement series. Almost the entire right side of the vehicle has been damaged. The apparent deformation was between the right $\mathrm{A}$ and $\mathrm{C}$ pillars and both vehicle doors were dented inside the vehicle. Furthermore, the right $\mathrm{B}$ pillar and the right doorsill (at the B pillar level) were broken.

When subtracing the deformations of the S3 impact using the top-view of the photograph (Figure 14 - up), the maximum deformation was about $26 \mathrm{~cm}$ and the minimum of $6 \mathrm{~cm}$. The three-dimensional analysis was again performed at the maximum deformation level and subsequently confirms that the impact was really striking (the greatest). The cut was measured at a height of $50 \mathrm{~cm}$ above the ground, the maximum deformation is was around $42 \mathrm{~cm}$ and a minimum about $10 \mathrm{~cm}$.

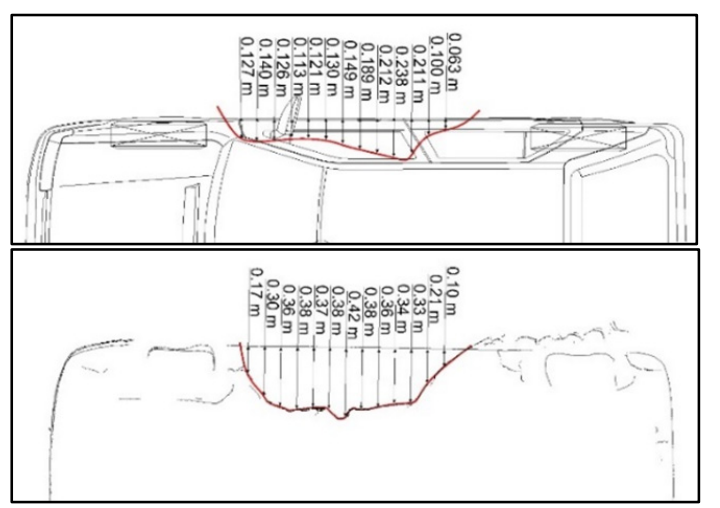

Figure 14: Skoda Felicia side impact - top-view (up) vs. scanner (down).

The case of side impact of S3 and the subsequent comparison of the two measurement methods shows that in the case of significant deformation, there are significant deviations between these methods as its shown in the graph below. Similarly as during analysis of F3 impact, this deviation can be eliminated by combination of top-view photography with another method of deformation measurement (e.g. levelling rod, meter, crush jigs, etc.). Case S3 also illustrate limitation of using this methodology for analysis of deformation from 3D scanner. Using only one $2 \mathrm{D}$ cut in the maximum deformation level can lead to inaccuracy in local maximum - e.g. in point 7 of S3 is the level of deformation higher in comparison with the other points. The maximum value could be caused e.g. by local crack. To achieve precise values and eliminate local errors would be more appropriate to use average of multiple sections (2D cuts).

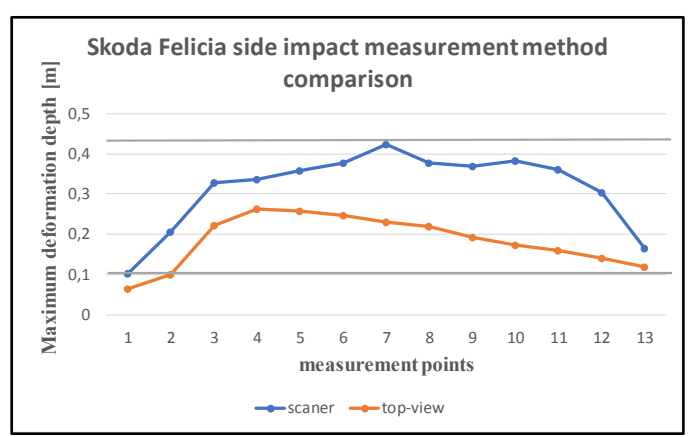

Figure 15: Skoda Felicia side impact measurement method comparison. 


\subsection{Comparison of Selected Methods Accuracy}

The deformation depth at the measured points was subsequently evaluated and plotted. From the graphs below, it is evident that in lateral impacts (Figure 16), the depth of deformation up to $10 \mathrm{~cm}$ is within a slight but acceptable tolerance. As the depth of deformation increases, so does the deviation from the ideal tendency. In some cases, it is up to $50 \%$ deviation, especially in the area of maximum deformation depth. There are several limitations of top-view photography. The accuracy of the obtained data is significantly influenced by the quality of photography, very important influence has been also the angle of taken photo. In cases where deformation interferes to the vehicle interior, the top-view photography method is not applicable for deformation analysis. Therefore, when evaluating these types of deformation, a combination of measurement methods must be considered to achieve higher measurement accuracy.

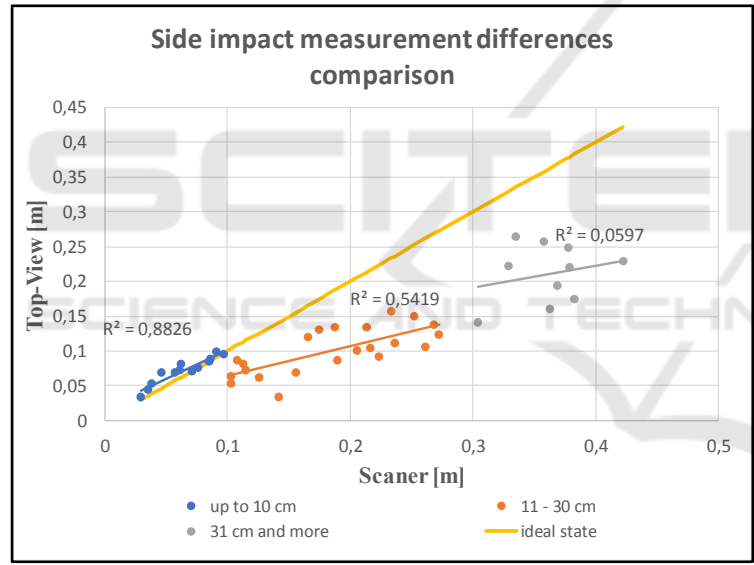

Figure 16: Side impact measurement differences comparison.

In the case of frontal impact and subsequent comparison of the measurement methods, the deviation is significantly lower compared to the side impact. With increasing depth of deformation, the deviation is higher but not as significant as in side impacts. But even here it is necessary to consider the so-called hidden deformations, which may not be obvious at first sight. These are, for example, deformations occurring at lower velocities, where elastic deformation is largely occurring plastic deformation is "hidden". The elasticity of the material has an overall effect on the measurement of the deformation depth (regardless of the type of used method), the measured values will be lower compared to the real vehicle damage.

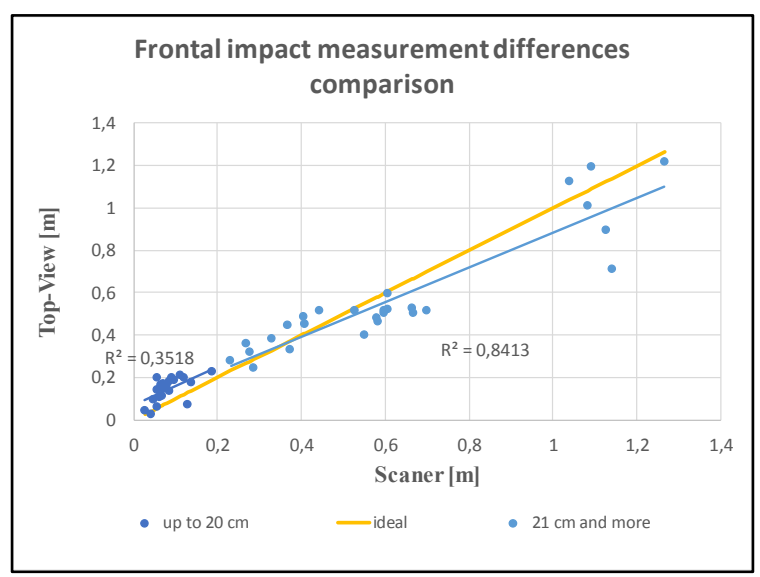

Figure 17: Frontal impact measurement differences comparison.

The basic statistical parameters were analysed to compare the methods of measuring the deformation depth. The deviation of the vehicle frontal deformation measurement is given in the table below (Table 1). The average deviation of the measurement increases with the depth of the deformation and ranges up to about $10 \mathrm{~cm}$. The maximum deviation is around $43 \mathrm{~cm}$, this significant deviation being found at F3 (see figures 8 and 9), in this case the deformation depth was covered by the windshield of the vehicle by using the top-view method. As mentioned above, this inaccuracy can be eliminated by using multiple methods.

Table 1: Frontal impact measurement deviation comparison.

\begin{tabular}{|c|c|c|c|}
\hline deviation & Mean $[\mathrm{cm}]$ & $\begin{array}{c}\text { Minimum } \\
{[\mathrm{cm}]}\end{array}$ & $\begin{array}{c}\text { Maximum } \\
{[\mathrm{cm}]}\end{array}$ \\
\hline to $10 \mathrm{~cm}$ & 7,1 & 0,8 & 14,2 \\
\hline from $11 \mathrm{~cm}$ & 9,4 & 1,1 & 42,6 \\
\hline
\end{tabular}

In case of lateral deformation (Table 2), the measurement deviation increases substantially. The average deviation up to $10 \mathrm{~cm}$ of the deformation depth is about $1 \mathrm{~cm}$, above $11 \mathrm{~cm}$ this deviation increased. Increasing deviation is evident in all measurement cases (S1, S2 and S3). Above $31 \mathrm{~cm}$, the average deviation is about $15 \mathrm{~cm}$ and maximum up to $21 \mathrm{~cm}$. This is the case of S3 (see Figure 15), where a significant deformation of the vehicle interior occurred, therefore it was not possible to identify the real deformation extent using only the top-view photography. 
Table 2: Side impact measurement deviation comparison.

\begin{tabular}{|c|c|c|c|}
\hline deviation & Mean [cm] & $\begin{array}{c}\text { Minimum } \\
{[\mathrm{cm}]}\end{array}$ & $\begin{array}{c}\text { Maximum } \\
{[\mathrm{cm}]}\end{array}$ \\
\hline to $10 \mathrm{~cm}$ & 0,8 & 0,0 & 2,2 \\
\hline from 11 to $30 \mathrm{~cm}$ & 8,5 & 2,2 & 15,5 \\
\hline from $31 \mathrm{~cm}$ & 15,1 & 7,1 & 20,8 \\
\hline
\end{tabular}

\section{DISCUSSION}

The aim of this study was to compare selected methods (top-view photography as basic and cheap method and 3D scanning as modern and advanced method) with respect to the achieved accuracy of deformation depth values as one of the basic parameters for the analysis of the accident. Similar methodology was used by Randles (2010) for the comparison of the photogrammetry and hands-on measurement. Different vehicles and 2 basic types of damage - front and side impact - were chosen for the analysis. Also, the different range of vehicle deformation depth were selected. Differences of the deformation depth measurement using scanning and top-view photography were illustrated by Figure 16 and Figure 17.

Obtained results illustrate not significant differences between the selected methods used for the analysis of frontal impact. Inaccuracy could be caused if the vehicle deformation has been covered by some of the vehicle parts (e.g. bonnet, bumper). This is however limitation of most of the used methods. Similar results have been proved also by some of the previous studies - e.g. (Boddorff, 1990). As stated by Comeau or Boddorf (Comeau, 1996; Boddorff, 1990), more accurate results could be achieved by understanding of the methods for accident reconstruction and documentation limitation. These conclusions illustrate the importance of these type of studies.

The highest deviations of the obtained results were related to the analysis of side impact. The usage of top-view photography has been very limited in case of side impact. While there are no significant deviations in the case of minor damage (up to $10 \mathrm{~cm}$ ), bigger deformation depth (from $20 \mathrm{~cm}$ ) shows significant inaccuracies when using top-view photography. It is therefore necessary to use a combination of several methods in such cases. The use of modern methods such as 3D scanning allows to achieve accurate results. The biggest disadvantage of three-dimensional methods has been the high purchase price and especially in case of photogrammetry the lack of time at the accident scene. (Massa a Barrette, 1998; Terpstra 2019).
Three-dimensional methods could serve not only for the analysis of vehicle damage but also can provide a comprehensive view of the whole accident site. (Coleman 2015; Callahan, 2012; Terpstra 2019).

The aim of the follow-up studies will be the comparison of some of the other commonly used methods for the deformation depth determination (photogrammetry, total geodetic station, crash jigs, etc.). The methods will be evaluated with respect to the achieved accuracy, but also in relation to the type of vehicle damage or the extent of vehicle deformation respectively. Most of the previous studies used for the analysis of the methods accuracy simulated accident scenario (e.g. Randles, 2010; Gaffney, 2015; Castaneda, 2012). As one of the main advantages of the study could be mentioned that not only the deformation of the vehicles damaged during crash tests or simulated accident scenarios were analysed, but also the real accidents. Working on the scene of real accidents has certain specifics compared to crash tests - especially time pressure due to road closure and related congestion, the accident participant presence on spot, etc. These factors could significantly affect the achieved accuracy and thus distort the obtained results. In the future work, the comprehensive assessment will be carried out with the aim to create the methodology for vehicle deformation documentation with respect to the extent and type of the vehicle damage.

\section{CONCLUSIONS}

With increase of the traffic intensity also the number of accidents increases. The traffic accident occurrence is associated with society-wide losses not only directly from the accident itself but also in relation to the congestion due to the road closures at the accident sites. The duration of the accident site documentation as well as vehicle deformation documentation has been important parameter. The time pressure during accident documentation may subsequently resulted to the insufficient documentation of the accident site and vehicles as well as the overall data quality (Topolšek 2013). The financial demand of the acquisition and data storage requirements are also necessary to take into account.

The aim of this article was to determine the applicability of selected methods depending on their accuracy in relation to the various types of collisions and extent of vehicle damage. On the basis of the selected results could be established that for the frontal impacts is mostly sufficient to use conventional methods. These methods are also 
suitable for small deformation (up to 10 centimetres). For the purpose of the side impacts and large deformation depth it is necessary to use modern documentation methods (e.g. scanner) or at least the combination of several methods.

\section{ACKNOWLEDGEMENTS}

This article was produced with the financial support of the Ministry of Transport within the programme of long-term conceptual development of research institutions on the research infrastructure acquired from the Operation Programme Research and Development for Innovations (CZ.1.05/2.1.00/ 03.0064).

\section{REFERENCES}

Brach, M., \& Brach, R. M. (1998). Crush Energy and Planar Impact Mechanics for Accident Reconstruction. SAE Technical Paper Series. doi:10.4271/980025

Campbell, K. L. (1974). Energy Basis for Collision Severity. SAE Technical Paper Series. doi:10.4271/740565

McHenry, R. R., \& McHenry, B. G. (1986). A Revised Damage Analysis Procedure for the CRASH Computer Program. SAE Technical Paper Series. doi:10.4271/861894

Brown, D. R., Wiechel, J. F., Stansifer, R. L., \& Guenther, D. A. (1987). Practical Application of Vehicle Speed Determination from Crush Measurements. SAE Technical Paper Series. doi:10.4271/870498

Tumbas, N. S., \& Smith, R. A. (1988). Measuring Protocol for Quantifying Vehicle Damage from an Energy Basis Point of View. SAE Technical Paper Series. doi:10.4271/880072

Boddorff, T. C. and Jones, I. S., "Simple Overhead Photography Techniques for Vehicle Accident Reconstruction," SAE Technical Paper 900370, 1990.

Comeau, J.-L., Dalmotas, D. J., German, A., Monk, B., \& Trépanier, D. (1996). Crush Measurement for Side Impacts Using a Total Station. SAE Technical Paper Series. doi:10.4271/960100

Massa, D. J., \& Barrette, R. W. (1998). Using ThreeDimensional Digitization to Model a Vehicle. SAE Technical Paper Series. doi:10.4271/980377

Terpstra, T., Dickinson, J., Hashemian, A., and Fenton, S., "Reconstruction of 3D Accident Sites Using USGS LiDAR, Aerial Images, and Photogrammetry," SAE Technical Paper 2019-01-0423, 2019

Coleman, C., Tandy, D., Colborn, J., \& Ault, N. (2015). Applying Camera Matching Methods to Laser Scanned Three Dimensional Scene Data with Comparisons to Other Methods. SAE Technical Paper Series. doi:10.4271/2015-01-141
Tumbas, Nicholas, S., Kinney, J., Rolly, Smith, C., Gregory, C., "Photogrammetry and Accident Reconstruction: Experimental Results," SAE 940925, 1994

Fenton, Stephen, Kerr, Richard, "Accident Scene Diagramming Using New Photogrammetric Technique", SAE 970944, 1997

Neale, W. T. C., Fenton, S., McFadden, S., \& Rose, N. A. (2004). A Video Tracking Photogrammetry Technique to Survey Roadways for Accident Reconstruction. SAE Technical Paper Series. doi:10.4271/2004-01-1221

Callahan, M., LeBlanc, B., Vreeland, R., and Bretting, G., "Close-Range Photogrammetry with Laser Scan Point Clouds," SAE Technical Paper 2012-01-0607, 2012

Randles, B., JoneS, B., Welcher, J., Szabo, T. et al., "The Accuracy of Photogrammetry vs. Hands-on Measurement Techniques used in Accident Reconstruction," SAE Technical Paper 2010-01-0065, 2010

Gaffney, T., Winter, B., Elston, A., Sandvik, A. et al., "Method for Estimating Vehicle-Specific Frontal Stiffness Values in the Absence of an Applicable Crash Test Using Methodically-Distilled Data from the NHTSA Crash Database (Phase 1)," SAE Technical Paper 2015-01-0027, 2015, doi:10.4271/2015-01-0027

Harrington, S., \& Lebak, G. (2018). The Placement of Digitized Objects in a Point Cloud as a Photogrammetric Technique. SAE International Journal of Transportation Safety, 6(2). doi:10.4271/0906-02-0007

Karczewski, M.. Influence of 3D scanner parameters on accuracy evaluation of vehicle element deformation. In: AIP Conference Proceedings, 2019, 2078(1). DOI: 10.1063/1.5092015.

Kang, H. J. a I. Han. 3-Dimensional Scanning/Modeling for Analysis of Vehicle Collision Accidents. Transactions of the Korean Society of Automotive Engineers. 2017, (25), pp. 541-547. DOI: 10.7467/KSAE.2017.25.5.541.

Buck, U., Naether, S., Braun, M., Bolliger, S., Friederich, H., Jackowski, C., ... Thali, M. J. (2007). Application of $3 \mathrm{D}$ documentation and geometric reconstruction methods in traffic accident analysis: With high resolution surface scanning, radiological MSCT/MRI scanning and real data based animation. Forensic Science International, 170(1), 20-28. doi:10.1016/j.forsciint.2006.08.024

Kamnik, R., Nekrep Perc, M., \& Topolšek, D. (2020). Using the scanners and drone for comparison of point cloud accuracy at traffic accident analysis. Accident Analysis \& Prevention, 135, 105391. doi:10.1016/j.aap.2019.105391 